\title{
Now Trending in Your Community: Social Media Insights For Your Public Health Mission
}

\author{
Diana M. Kushner* \\ Department of Health and Human Services, Washington, DC, USA
}

\section{Objective}

The goal of the Now Trending website is to provide a web based tool that pulls out relevant Twitter conversations concerning illness and disasters and provides meaningful analytics on how those conversations are trending. The website gives the user the ability to view trends overall and for specific geographic areas.

\section{Introduction}

In today's fast paced world, information is available (and expected) instantaneously. Social media has only fueled this expectation as it has permeated all aspects of our lives. More and more of the population is turning to social media outlets to share their thoughts and update their status, especially during disasters. With all these conversations occurring, it is only reasonable to assume that health status is part of the information being shared. In fact, studies by Johns Hopkins University $^{1}$ and Harvard University ${ }^{2}$ have shown that social media reporting can serve as an early indicator and warning of emerging health issues within a community. Whether people are talking about being sick themselves or fear of illness in the community, there is a wealth of knowledge to be gained by tapping into this information. Yet gaining insight and understanding from social media data can be problematic. The unstructured nature of the data, the presence of social media "spam", and the frequency of reposting information makes social media a noisy data source. Being able to harness this data would provide the opportunity to use social media as an effective situational awareness and early warning tool for biosurveillance missions. But how do you accomplish this? There are tens of millions of conversations happening on social media every day that would need to be sifted through to get to the health related topics. No public health entity has the time or staffing for that endeavor.

\section{Methods}

Utilizing an innovation challenge, the Assistant Secretary for Preparedness and Response (ASPR) within the Department of Health and Human Services held a competition to create a web-based application that would provide federal, state, and local public health authorities the ability to cut through the vast amounts of Twitter data and pull out relevant conversations on illness for the national level as well as their geographic area of interest. This information is then available to use in multiple ways such as serving as an indicator of potential health issues emerging in the population, building a baseline of trend data, engaging the public on trending health topics, or crossreferencing other data sources. Working with the winning team, ASPR transitioned the winning website over to government cloud space to provide consistent and reliable tool for its public health partners. This website, https://nowtrending.hhs.gov, is available free of charge to not only public health practitioners, but all interested parties.

\section{Results}

The Now Trending application is a web-based tool which allows users to review trends in discussions of illness and disaster related topics on Twitter in real time. Users of Now Trending have the ability to view analytics of Twitter trends by geographic area or by health condition. The analytics are shown as time period trends which range from the previous 2 hours to the previous week. Users can click on points in the graph to see the actual tweets that make up the trends in order to gain better context of the Twitter conversations.

Now Trending cuts through the noise in Twitter by filtering health condition searches using a list of terms for each health condition, including the common terms used by the general population and some of the common symptoms associated with the condition. In addition, Now Trending uses qualifier terms to further eliminate irrelevant Tweets. There are currently 27 health conditions tracked by Now Trending, but this can be easily expanded to include terms that are relevant to current events.

\section{Conclusions}

The Now Trending website is being presented to ensure all public health personnel are aware of this free tool and the advantages it can provide them in their public health missions.

\section{Keywords}

Social Media; Biosurveillance; Geolocation

\section{References}

[1] Paul, Michael and Dredze, Mark. You Are What You Tweet: Analyzing Twitter for Public Health. Baltimore: Johns Hopkins University, 2011.

[2] Keller, Mikaela, et al. "Use od Unstructured Event-Based Reports for Global Infectious Disease Surveillance." Emerging Infectious Disease 15, no. 5 (May 2009): 689-695.

*Diana M. Kushner

E-mail: diana.kushner@hhs.gov 